\title{
Giant Scalp Melanoma
}

\author{
${ }^{1}$ Emine Böyük, ${ }^{1}$ Issıl Bulur, ${ }^{1}$ Zeynep Nurhan Saraçoğlu, ${ }^{2}$ Özlem Erdem \\ ${ }^{1}$ Department of Dermatology, Eskişehir Osmangazi University, Faculty of Medicine, Eskişehir, \\ ${ }^{2}$ Department of Pathology, Gazi University, Faculty of Medicine, Ankara, Turkey \\ "email: emineboyuk@gmail.com
}

To the Editor,

A 54-year-old female presented with a 4month history of extensive dark-colored lesions on her scalp. The pathology result of a previous biopsy from her scalp performed by a general surgeon a year ago had been congenital melanocytic nevus. Dermatological examination showed confluent blue-grey, dark brown macules, papules and nodules covering most of the left parietofrontotemporal scalp in an area measuring $15 \times 30 \mathrm{~cm}$ in size (Figure 1a). There were also satellite lesions of varying diameters on the ear, anterior trunk and one pigmented lesion of the oral mucosa. Dermoscopic analysis of the lesions revealed asymmetry of color and structure, multicomponent global pattern, irregular dots and globules and bluish-white color (Figure $1 b)$.

Histopathologic examination of three 4-mm punch biopsies from the patient's scalp revealed atypical melanocytic lesions with findings that were not adequate to confirm melanoma. Total excision of the scalp lesion was not approved by plastic surgery. The total excision material of the pigmented lesion on her anterior trunk was referred to another pathologist and revealed superficial spreading melanoma with Breslow thickness of $1 \mathrm{~mm}$, Clark level of 3 and mitotic rate of $2 / \mathrm{mm}^{2}$ (Figure 1c). In addition, pulmonary metastases were detected by total body positron emission tomography-computed tomography (PET-CT). The case was accepted as T1N3M1 inoperable metastatic malign melanoma originating from the scalp lesion. Vemurafenib treatment was started by medical oncology. However, the patient developed nodules and ulcerations, cervical lymph node metastasis and vena cava superior syndrome during the treatment and died nine months after the diagnosis.

Giant melanoma is a rare presentation of cutaneous melanomas. A literature search revealed 18 previous patients with giant melanoma with only three originating from the scalp (1-4).

Most giant melanoma cases have no precursor lesion but the lesion developed in a congenital nevus in two cases and from a previous melanocytic nevus in another two cases (5). No precursor lesion was found in three cases with giant scalp melanoma $(1,3,4)$. On the other hand, our patient described a pigmented lesion on her scalp since childhood and a biopsy from the lesion had revealed a congenital melanocytic nevus.

Giant melanomas usually present as a vegetative tumor with high Breslow thickness. Two of the three patients with a giant scalp melanoma in the literature presented with a vegetative tumor, and the other one presented 
with confluent purple-blue-gray macules, papules and nodules as in our patient $(1,3,4)$.

Excisional biopsy from the anterior trunk in our case revealed superficial spreading melanoma with a Breslow thickness of $1 \mathrm{~mm}$ while three punch biopsies from the scalp were not sufficient to confirm melanoma. The punch biopsy method might not be helpful in the diagnosis of some giant melanomas, probably due to sampling errors. Dermoscopy is also generally inadequate in identifying the suspicious areas in these cases. We therefore recommend a large incisional biopsy or excisional biopsy instead of punch biopsy in giant melanomas to avoid delays in diagnosis.

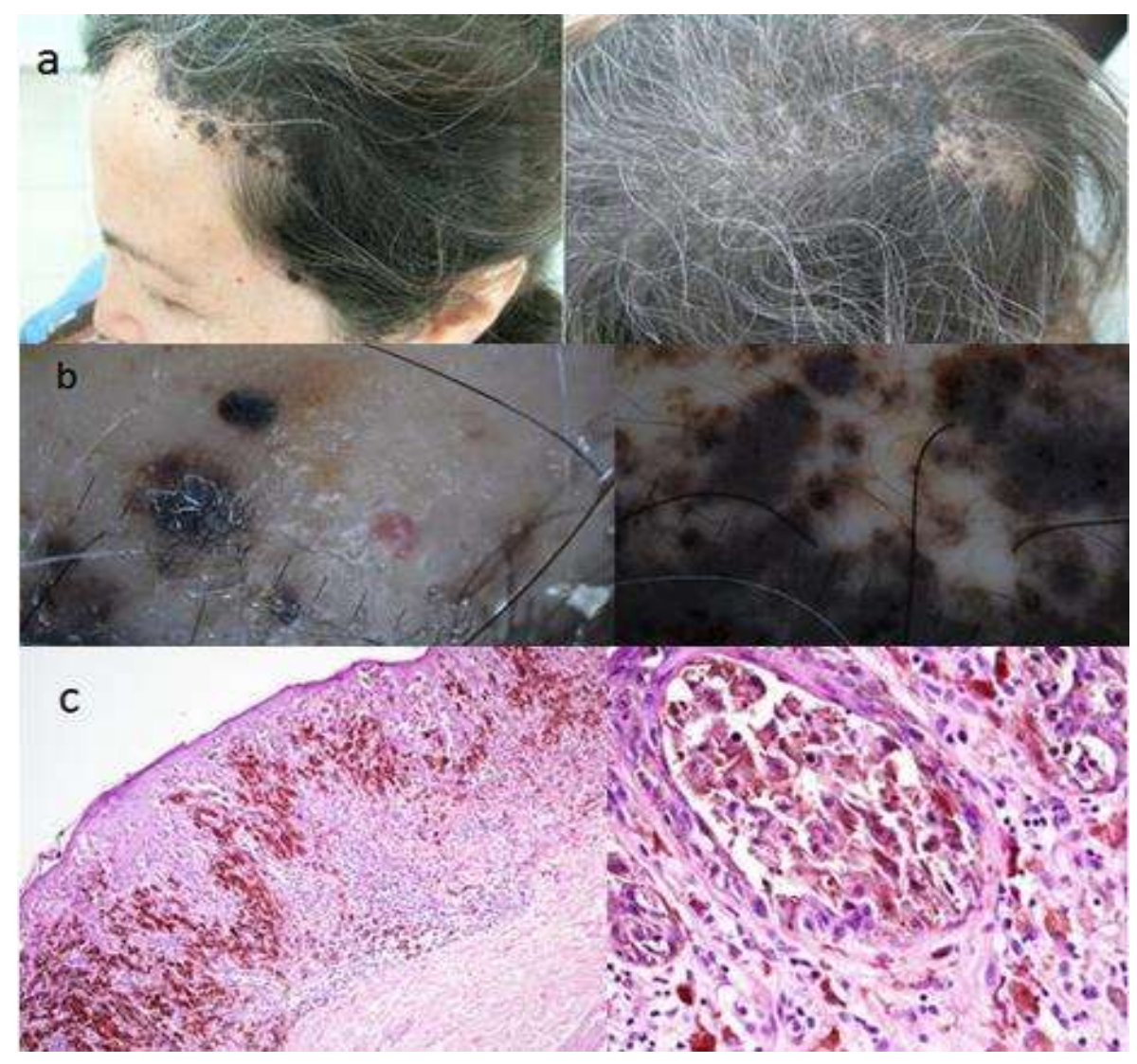

Figure 1: (1a).Clinical presentation of Giant scalp malign melanoma at diagnosis, (1b). Dermoscopic image of the lesions: multicomponent global pattern, irregular dots and globules and bluish-white color, (1c). Histopathological examination, atypical melanocytic cell infiltration in the epidermis and dermis, a) $H \& E x 100, b) H \& E x 200$.

\section{REFERENCES}

1. Ching, J. A., \& Gould, L. (2012). Giant scalp melanoma: a case report and review of the literature. Eplasty, 12.

2. Martínez-Cabriales, S. A., Ayala-Cortés, A. S., Vázquez-Martínez, O. T., Welsh-Lozano, O., Miranda-Maldonado, I., \& Ocampo-Candiani, J. (2014). A neglected giant metastatic melanoma. Journal of the European Academy of Dermatology and Venereology.

3. Müller, C. S., Hinterberger, L., Vogt, T., \& Pföhler, C. (2011). Giant melanoma of the scalp-discussion of a rare clinical presentation. BMJ case reports, 2011, bcr1220103643.
4. Panajotovic, L., Đordevic, B., \& Pavlovic, M. D. (2007). A giant primary cutaneous melanoma of the scalp-can it be that big?. Journal of the European Academy of Dermatology and Venereology, 21(10), 1417-1418.

5. di Meo, N., Stinco, G., Gatti, A., Errichetti, E., Bonin, S., Albano, A., \& Trevisan, G. (2014). Giant melanoma of the abdomen: case report and revision of the published cases. Dermatology online journal, 20(7). 